\title{
Turbo Equalisation for the Enhanced GPRS System
}

\author{
Markku Pukkila \\ Nokia Research Center \\ P.O. Box 407, FIN-00045 Nokia Group, Finland \\ E-mail: markku.pukkila@nokia.com
}

\begin{abstract}
The iterative receiver structures can often improve the performance with a reasonable level of complexity. In this paper, we study iterative equalisation technique, i.e., turbo equalisation (TE), which enables to improve the performance by signal processing means in the receiver - without altering any other part of the system. TE is based on the feedback information from the channel decoder into the equaliser. That information is used as a priori information in the equaliser to start a new iteration, which provides more reliable decisions. The performance evaluation for Enhanced General Packet Radio System (EGPRS) shows that TE is beneficial for 8-PSK modulation in particular giving $2 \mathrm{~dB}$ gain with one extra iteration. However, TE requires soft-in-softout (SISO) decoder to be used, since the feedback information from the decoder should contain reliability information.
\end{abstract}

\section{INTRODUCTION}

Turbo coding techniques has been studied extensively since published by Berrou et al. in 1993 [1], since they can improve the receiver performance significantly by a relatively simple iterative receiver structure. The same principle can be modified to iterative equalisation - or turbo equalisation (TE) - by considering the multipath transmission channel as a convolutional code of rate 1 . Thus the equalisation and decoding can be performed in an iterative fashion so that the equaliser exploits the extrinsic information obtained from the previous decoding round. As a result, the decisions become more reliable and the receiver performance improves.

A number of researchers has been studied TE as well. In $[2,3]$ the TE principle is analysed in detail concerning e.g., how the extrinsic information should be composed and used during the iteration. In [4] TE is studied for GSM Speech Services and [6] gives an analysis for the EDGE (Enhanced Data Rates for GSM Evolution) system. Different SISO decoders are compared in [5] for the TE purposes.

In the EDGE system, the 8-PSK modulation complicates the receiver algorithms due to the multilevel transmission symbols. Since the number of trellis states in the equaliser grows exponentially, the full Maximum Likelihood Sequence Estimation (MLSE)

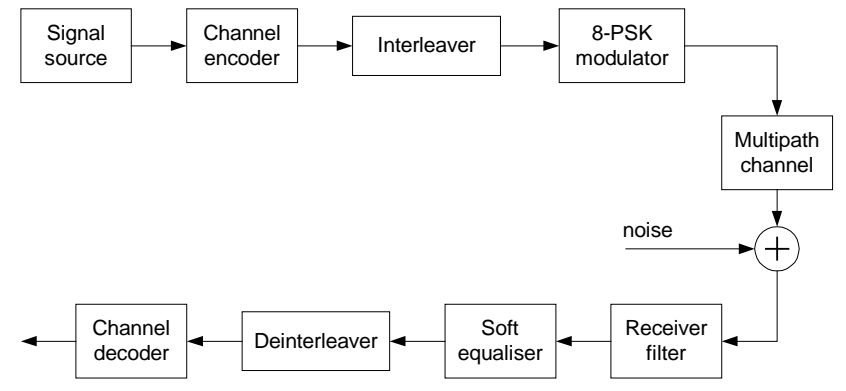

Figure 1. Transmission system model.

cannot be implemented, but usually some suboptimum decision feedback technique like Decision Feedback Equaliser (DFE) or Decision Feedback Sequence Estimation (DFSE) is chosen. Therefore it is attractive to compensate the performance loss by the iterative TE method.

This paper is organised as follows. First we describe the transmission system model for the EGPRS system with the 8-PSK modulation. After that the principle of TE is depicted in detail. Then we describe the receiver algorithms, i.e., the DFSE equaliser and max-logMAP (Maximum A Posteriori) channel decoder algorithms. The receiver performance is evaluated by computer simulations in several Rayleigh fading channels and finally conclusions are drawn.

\section{SYSTEM MODEL}

In this paper, we use the EGPRS platform, which is modelled in Fig. 1. In the transmitter side, a block of data bits $\mathbf{u}$ is protected by a convolutional encoder and interleaved over four successive transmission bursts to overcome fast fading phenomenon. The encoded bits $\mathbf{c}=\left(c_{0}, c_{1}, \ldots, c_{3 K-1}\right)^{T}$ are modulated using 8-PSK alphabet, i.e., every 3 bits form a single 8-PSK symbol. Those symbols are denoted by $\mathbf{a}=\left(a_{0}, a_{1}, \ldots, a_{K-1}\right)^{T}$. The modulated signal is transmitted over a frequency selective fading channel. In this paper, we assume block fading channel characteristics, hence the channel is time-invariant during one transmission burst. Thermal noise at the receiver is modelled as additive white Gaussian noise (AWGN).

The received signal $\mathbf{r}$ that is sampled at the symbol rate can be given by the equation

$$
\mathbf{r}=\mathbf{A h}+\mathbf{w}
$$


where $\mathbf{A}$ is the matrix containing symbols $a_{k}$ as follows

$$
\mathbf{A}=\left[\begin{array}{cccc}
a_{L} & a_{L-1} & \cdots & a_{0} \\
a_{L+1} & a_{L} & & a_{1} \\
\vdots & & \ddots & \vdots \\
a_{K+L-1} & a_{K+L-2} & \cdots & a_{K-1}
\end{array}\right]
$$

The channel impulse response is described by the vector $\mathbf{h}=\left(h_{0}, h_{1}, \ldots, h_{L}\right)^{T}$, which consists of symbolspaced complex-valued channel taps. The white noise samples are denoted by $\mathbf{w}$; the noise variance is $\sigma^{2}=N_{0} / 2$.

First of all the equaliser needs to estimate the channel impulse response by using the known training (midamble) symbols in the middle of the burst. The maximum-likelihood (ML) channel estimate in the presence of white noise is given by [7]

$$
\hat{\mathbf{h}}=\left(\mathbf{M}^{H} \mathbf{M}\right)^{-1} \mathbf{M}^{H} \mathbf{r}
$$

where $\mathbf{M}$ is the training sequence matrix formed from the midamble symbols according to (2).

Due to the multilevel 8-PSK symbols, the number of signal states in the full maximum likelihood sequence estimation by the Viterbi algorithm (MLSE-VA) would be far too many, so we need to use a less complex equaliser like DFE or DFSE. Both of them incorporate the previous symbol decisions in the metrics calculation and therefore the number of states is significantly reduced.

The equaliser provides soft outputs, i.e., reliability information on the coded bits for the channel decoder. The soft information on the bit $c_{\mathrm{k}}$ is usually given as a log-likelihood ratio (LLR) or $L$-value

$$
\lambda^{E}\left(c_{k}\right)=\log \frac{P\left(c_{k}=+1 \mid \mathbf{r}\right)}{P\left(c_{k}=-1 \mid \mathbf{r}\right)},
$$

which is the ratio between the conditional bit probabilities in the logarithmic domain. These $L$-values are deinterleaved and given for the channel decoder, which uses them to recover the information bits $\mathbf{u}$.

\section{PRINCIPLE OF TURBO EQUALISATION}

The previous chapter described the conventional system, but let us consider in more detail the iterative receiver structure, which is presented in Fig.2. In this paper we assume that the channel state information is either known or the provided estimate is constant over the whole iteration process. In [15] we consider an iterative channel estimation scheme, where the feedback information is also utilised in the estimation of the channel impulse response.

At the first iteration round there is no feedback information from the channel decoder available, so the equaliser calculates the $L$-values $\lambda^{E}\left(\mathbf{c}^{\prime}\right)$ as given by (4) that are just based on the received samples $\mathbf{r}$ from the

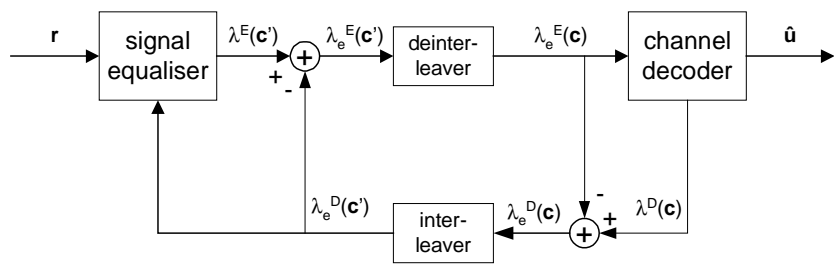

Figure 2. Turbo equaliser structure.

channel. The $L$-values are deinterleaved to break consecutive bits far apart and thus giving the channel decoder independent input values. The interleaving is an essential part in the iterative receiver scheme, since the extra information on an individual data bit is due to the different neighbouring bits in the detection and decoding processes.

The soft values $\lambda^{E}(\mathbf{c})$ are provided for the SISO channel decoder. The decoder has to calculate new $L$ values $\lambda^{D}(\mathbf{c})$ for the coded bits $\mathbf{c}$, since they are needed in the feedback branch to the equaliser. Therefore we need to use the more complex SISO decoder instead of the conventional hard output decoder.

The equaliser is able to produce the $L$-values $\lambda^{E}(\mathbf{c})$ based on the received samples from the channel, so that information should not be repeated in the feedback. Hence, the feedback only contains the extra information that is obtained from the surrounding bits in the channel decoding. The input $L$-values and the obtained extra information are called intrinsic and extrinsic information, respectively. The extrinsic information from the channel decoder is given as [2,3]

$$
\lambda_{e}^{D}\left(c_{k}\right)=\lambda^{D}\left(c_{k}\right)-\lambda_{e}^{E}\left(c_{k}\right),
$$

where $\lambda_{e}^{E}\left(c_{k}\right)$ denotes the extrinsic information from the equaliser.

The turbo equalisation technique is based on the utilisation of this extrinsic information at the next iteration round [2]. So it is passed through the interleaver to the equaliser as a priori information on the bit reliabilities. By exploiting this side information in the detection, more reliable decisions are achieved. Also in the equaliser output the extrinsic information $\lambda_{e}^{E}\left(c_{k}\right)$ is extracted from the output as follows

$$
\lambda_{e}^{E}\left(c_{k}\right)=\lambda^{E}\left(c_{k}\right)-\lambda_{e}^{D}\left(c_{k}\right) .
$$

This equaliser information is again used in the SISO decoder to produce new soft outputs and furthermore, the new extrinsic information according to (5). As soon as this feedback information becomes available, the new iteration round can be started.

The number of iterations may depend on the processing power available or the achieved performance improvement. At the final stage, there is no need for the 
SISO decoder, since only hard decisions $\hat{\mathbf{u}}$ on the information bits are needed.

The TE receiver is able to improve the performance, but at the cost of higher complexity. The main burden is the complex SISO decoder, especially due to the EGPRS coding schemes that are based on the constraint length of 7. Also, as the equalisation and decoding are performed several times, the receiver complexity grows respectively.

In the next chapter, we describe in more detail DFSE (for signal equalisation) and max-log-MAP algorithm (for channel decoding).

\section{RECEIVER ALGORITHMS}

\section{A. Decision-Feedback Sequence Estimation}

The number of signal states that MLSE-VA requires is $M^{L}$, where $M$ is the size of the modulation alphabet and $L$ is the length of the channel memory. Therefore, when multilevel modulations $(M>2)$ are considered, MLSE-VA becomes impractical due to the excessive trellis size. However, the MLSE problem can be recursively solved by the DFSE algorithm, which considers only $D$ most recent symbols in the trellis and the earlier $L-D$ symbols are used through the embedded decision-feedback structure [14]. Hence, the number of states can be reduced to much smaller amount of $M^{D}$.

The MLSE problem is to find the sequence of data symbols $\hat{\mathbf{a}}$ which maximises the likelihood function as follows [13]

$$
\hat{\mathbf{a}}=\underset{\mathbf{a}}{\arg \max } p(\mathbf{r} \mid \mathbf{a}) .
$$

An equivalent problem is to maximise the loglikelihood function $\log p(\mathbf{r} \mid \mathbf{a})$, which is solved by minimising the path metric

$$
P M=\|\mathbf{r}-\mathbf{A} \mathbf{h}\|^{2}=\sum_{k}\left(r_{k}-t_{k}\right)^{2}
$$

where

$$
t_{k}=\sum_{l=0}^{L} a_{k-l} h_{l} .
$$

In MLSE-VA the trellis is spanned by all the $L$ symbols in (9), but in DFSE the metric is composed from two parts so that (9) is replaced by [14]

$$
t_{k}=\sum_{l=0}^{D} a_{k-l} h_{l}+\hat{w}_{k-D-1}^{(s)},
$$

where $\hat{w}_{k-D-1}^{(s)}$ contains the feedback information saved in the current trellis state $s$. That information is extracted from the survival path $\hat{\mathbf{a}}^{(s)}$ that is leading to the state as follows

$$
\hat{w}_{k-D-1}^{(s)}=\sum_{l=D+1}^{L} \hat{a}_{k-l}^{(s)} h_{l}
$$

As a result, the trellis is spanned over $D$ symbols and the feedback information is updated for each state while the recursion proceeds.

The output from the equaliser is in the form of LLR (4), given in the presence of a priori information as follows

$$
\lambda^{E}\left(c_{k}\right)=\log \frac{P\left(c_{k}=+1 \mid \mathbf{r}\right) P\left(c_{k}=+1\right)}{P\left(c_{k}=-1 \mid \mathbf{r}\right) P\left(c_{k}=-1\right)},
$$

where the a priori information can be estimated from the extrinsic information [2]

$$
\log \frac{P\left(c_{k}=+1\right)}{P\left(c_{k}=-1\right)} \approx \lambda_{e}^{D}\left(c_{k}\right) .
$$

Combining (12) and (13) we obtain the output

$$
\lambda^{E}\left(c_{k}\right)=\log \frac{P\left(c_{k}=+1 \mid \mathbf{r}\right)}{P\left(c_{k}=-1 \mid \mathbf{r}\right)}+\lambda_{e}^{D}\left(c_{k}\right) .
$$

Since only the first few channel taps form the trellis structure, it is beneficial for DFSE that the channel is minimum phase, which is achieved by a separate feedforward transversal filter - or prefilter [14]. The prefilter can be designed with the same methods as the feedforward filter of the DFE receiver, e.g., by minimising the mean-squared error [8] or maximising the signal-to-noise ratio [9] in the input of the decision device.

\section{B. Soft-in-Soft-out Decoding}

The objective of the MAP algorithm is to minimise the bit error probability by estimating a posteriori probabilities (APP) of states and transitions of the Markov source from the received signal sequence. The MAP algorithm introduced by Chang and Hancock [10] uses the information of the whole received sequence to estimate a single bit probability. In other words, the MAP algorithm selects the bit $u_{k} \in\{-1,+1\}$ at time instant $k$, which maximises the following APP

$$
\hat{u}_{k}=\arg \max _{u_{k}}\left[p\left(u_{k} \mid \hat{\mathbf{c}}\right)\right] \text {. }
$$

The optimum MAP algorithm saves multiplicative transition probabilities in the trellis, which is computationally difficult. Therefore in practical implementations the path metric is calculated in the log-domain, which enables cumulative calculations [11,12]. Since MAP requires both forward and backward recursions, it is around two times more complex than the soft output Viterbi algorithm (SOVA) [12]. The main advantage of the MAP algorithm is more reliable soft information, since it is optimised for symbolwise decoding. This is why MAP is very suitable for the SISO decoding algorithm in the TE scheme. 
The BCJR-log-MAP (BCJR stands for Bahl, Cocke, Jelinek and Raviv) provides the APP information for each bit as the $L$-value according to (4). The state probability for trellis state $s$ at time $k$ is denoted by

$$
\alpha_{k}(s)=p\left(s, \mathbf{r}_{j \leq k}\right)
$$

in the forward direction and by

$$
\beta_{k}(s)=p\left(\mathbf{r}_{j>k} \mid s\right)
$$

in the backward direction. The transition probability between states $s$, and $s$ is given in log-domain as

$$
\ln \gamma_{k}\left(s^{\prime}, s\right)=\sum_{i=1}^{N} \frac{1}{2} \lambda_{e}^{E}\left(c_{k, i}\right) c_{k, i},
$$

where $c_{k, i}$ is the $i$ th code bit for the information bit $u_{k}$ and the coding rate is $1 / N$.

Then the decoder output is given as $[5,12]$

$$
\begin{gathered}
\lambda\left(\hat{u}_{k}\right)=\ln \sum_{\left(s^{\prime}, s\right)} e^{\ln \alpha_{k-1}(s)+\ln \gamma_{k}\left(s^{\prime}, s\right)+\ln \beta_{k}(s)} \\
-\ln \sum_{\substack{\left.u_{k}=+1 \\
s_{k}, s\right)}} e^{\ln \alpha_{k-1}\left(s^{\prime}\right)+\ln \gamma_{k}\left(s^{\prime}, s\right)+\ln \beta_{k}(s)}
\end{gathered}
$$

and the state probabilities can be computed recursively

$$
\begin{aligned}
& \ln \alpha_{k}(s)=\ln \sum_{s^{\prime}} e^{\ln \gamma_{k}\left(s^{\prime}, s\right)+\ln \alpha_{k-1}\left(s^{\prime}\right)} \\
& \ln \beta_{k}\left(s^{\prime}\right)=\ln \sum_{s} e^{\ln \gamma_{k+1}\left(s^{\prime}, s\right)+\ln \beta_{k+1}(s)} .
\end{aligned}
$$

To simplify the calculation the following approximation is used [12]

$$
\ln \left(e^{\delta_{1}}+\cdots+e^{\delta_{n}}\right)=\max _{i} \delta_{i}
$$

after which we achieve the form of BCJR-max-logMAP having the following output

$$
\begin{aligned}
\lambda\left(\hat{u}_{k}\right) & =\max _{\substack{\left(s^{\prime}, s\right) \\
u_{k}=+1}}\left\{\ln \alpha_{k-1}\left(s^{\prime}\right)+\ln \gamma_{k}\left(s^{\prime}, s\right)+\ln \beta_{k}(s)\right\} \\
& -\max _{\left(s^{\prime}, s\right)}\left\{\ln \alpha_{k-1}\left(s^{\prime}\right)+\ln \gamma_{k}\left(s^{\prime}, s\right)+\ln \beta_{k}(s)\right\}
\end{aligned}
$$

with

$$
\begin{aligned}
& \ln \alpha_{k}(s)=\max _{s^{\prime}}\left\{\ln \gamma_{k}\left(s^{\prime}, s\right)+\ln \alpha_{k-1}\left(s^{\prime}\right)\right\} \\
& \ln \beta_{k}\left(s^{\prime}\right)=\max _{s}\left\{\ln \gamma_{k+1}\left(s^{\prime}, s\right)+\ln \beta_{k+1}(s)\right\} .
\end{aligned}
$$

Finally, the soft information on the code bits $\lambda\left(c_{k}\right)$ (needed for the feedback) is obtained by re-encoding the achieved output (23).

\section{PERFORMANCE RESULTS}

In this chapter, the performance of the TE receiver in the EGPRS system is evaluated by simulations. Two coding schemes are studied, i.e., MCS-1 (GMSK modulation) and MCS-5 (8-PSK). Block error rates (BLER) with TU3 and HT100 channels are given for MCS-1 in Fig. 3 and 4 and for MCS-5 in Fig. 5 and 6. The given curves describe the performance after $1^{\text {st }}$ (which corresponds to the conventional receiver performance), $2^{\text {nd }}$ and $4^{\text {th }}$ iteration.

Small gains are achieved for MCS-1, i.e., only around $0.2 \mathrm{~dB}$ at BLER $10^{-2}$ after $2^{\text {nd }}$ iteration and totally 0.4 $\mathrm{dB}$ after $4^{\text {th }}$ iteration. Both channel types perform almost alike.

For MCS-5 better results are obtained. The gain after $2^{\text {nd }}$ iteration is $2.0 \mathrm{~dB}$ in TU3 and almost as much for HT100. These values are measured at BLER $10^{-1}$. Some extra improvement is still achieved by performing further iteration rounds; total gain after $4^{\text {th }}$ iteration is around $3.0 \mathrm{~dB}$. However, each new iteration increases the receiver complexity at the same time.

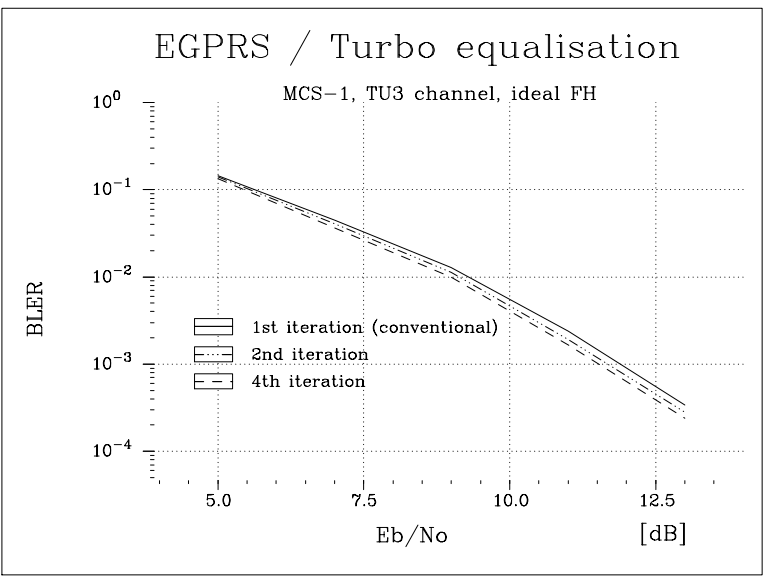

Figure 3. TE performance for MCS-1 (TU3).

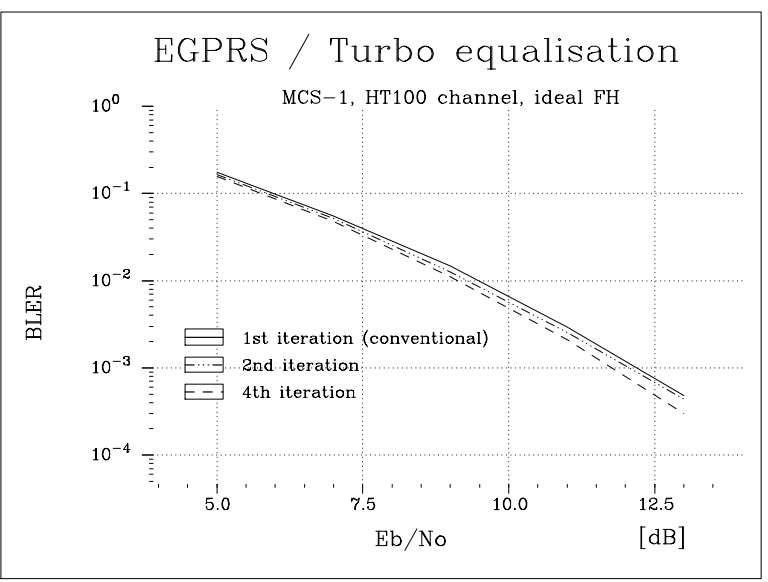

Figure 4. TE performance for MCS-1 (HT100). 


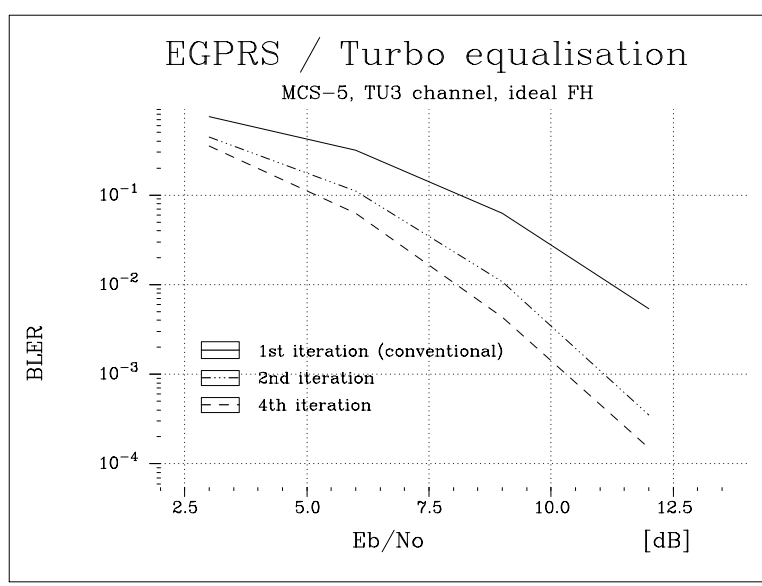

Figure 5. TE performance for MCS-5 (TU3).

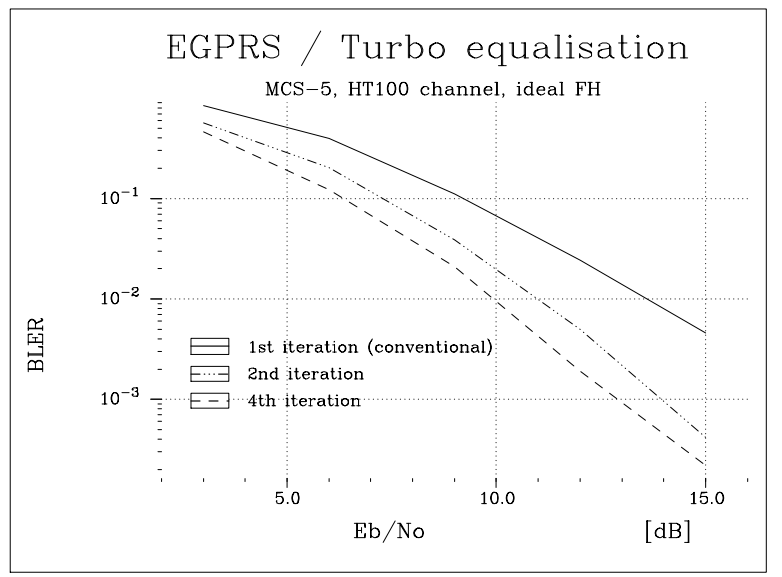

Figure 6. TE performance for MCS-5 (HT100).

\section{CONCLUSIONS}

Turbo equalisation could improve the receiver performance with a reasonable complexity increase. The feedback information from the channel decoder is exploited in the new iteration as a priori information in the equaliser and thus more reliable decisions are achieved. Most of the performance gain is usually obtained after a few iterations.

In this paper we study TE technique in the EGPRS system for both the 8-PSK and GMSK modulations. The communications system is described and the TE principle with the receiver algorithms are presented in more detail.

The performance is evaluated by simulations in the fading channels using ideal frequency hopping. MCS1 coding scheme exploits GMSK modulation, which can provide only around $0.2 \mathrm{~dB}$ improvement after the $2^{\text {nd }}$ iteration. Further iterations can provide another 0.2 $\mathrm{dB}$ gain on top of that. MCS-5 exploits 8-PSK modulation, which provides $2.0 \mathrm{~dB}$ gain in TU3 and almost the same in HT100. At least $1 \mathrm{~dB}$ extra gain is achievable by further iterations, but increasing the complexity at the same time. The TE algorithm also increases the complexity inherently due to the required SISO channel decoder.

\section{REFERENCES}

[1] C. Berrou, A. Glavieux and P. Thitimajshima, "Near Shannon Limit Error-Correcting Coding and Decoding: Turbo-Codes", Proc. ICC'93, Geneva, Switzerland, May 1993, pp. 1064-1070.

[2] C. Douillard, M. Jézéquel, C. Berrou, A. Picart, P. Didier and A. Glavieux, "Iterative Correction of Intersymbol Interference: Turbo-Equalization", European Trans. on Telecommunications, Vol. 6, No. 5, Sep-Nov 1995, pp. 507-511.

[3] A. Picart, P. Didier and A. Glavieux, "Turbo-Detection: A new approach to combat channel frequency selectivity", Proc. ICC'97, pp. 1498-1502.

[4] G. Bauch and V. Franz, "Iterative Equalization and Decoding for the GSM-System,” VTC'98, May 1998, pp. 2262-2266.

[5] G. Bauch and V. Franz, "A Comparison of Soft-In/Soft-Out Algorithms for "Turbo-Detection”, ICT98.

[6] P. Strauch, C. Luschi, M. Sandell and R. Yan, "Turbo Equalization for an 8-PSK Modulation Scheme in a Mobile TDMA Communication System", VTC'99, pp. 1605-1609.

[7] S. Kay, Fundamentals of Statistical Signal Processing: Estimation Theory, Prentice Hall, Vol I, New Jersey, 1998.

[8] J.G. Proakis, Digital Communications, McGraw-Hill, $3^{\text {rd }}$ Edition, New York, 1995.

[9] W.H. Gerstacker, J.B Huber, "Maximum SNR Decisionfeedback Equalization with FIR Filters: Filter Optimization and a Signal Processing Application", Proc. ICC'96, pp.1188-1192.

[10] R.W. Chang and J.C. Hancock, "On Receiver Structures for Channels Having Memory", IEEE Trans. on Inf. Theory, Vol. 12, No. 3, pp. 463-468, Oct. 1996.

[11] Y. Li, B. Vucetic, and Y. Sato, "Optimum Soft-Output Detection for Channels with Intersymbol Interference", IEEE Trans. on Inf. Theory, Vol. IT-41, No. 3, pp. 704-713, May 1995.

[12] P. Robertson, E. Villebrun, and P. Hoeher, "A Comparison of Optimal and Sub-Optimal MAP Decoding Algorithms Operating in the Log Domain", Proc. ICC'95, Seattle, WA, June 1822, 1995, pp. 1009-1013.

[13] G. D. Forney, Jr., "Maximum-likelihood Sequence Estimation of Digital Sequences in the Presence of Intersymbol Interference", IEEE Trans. Inf. Theory, Vol. IT-18, pp. 363-378, May 1972 .

[14] A. Duel-Hallen, and C. Heegard, "Delayed Decision-Feedback Sequence Estimation", IEEE Trans. Comm., Vol. 37, No. 5, pp. 428-436, May 1989.

[15] N. Nefedov, and M. Pukkila, "Iterative Channel Estimation for GPRS", published in PIMRC'2000, London, UK, Sep 2000. 